\title{
Decoupling in the Design and Synthesis of Multivariable Control Systems
}

\author{
PETER L. FALB, MEMbER, IEEE, AND WILLIAM A. WOLOVICH, MEMBER, IEEE
}

\begin{abstract}
Necessary and sufficient conditions for the "decoupling" of an $m$-input, $m$-output time-invariant linear system using state variable feedback are determined. Given a system which satisfies these conditions, i.e., which can be decoupled by state variable feedback, the class $\Phi$ of all feedback matrices which decouple the system is characterized. The characterization of $\Phi$ is used to determine the number of closed-loop poles which can be specified for the decoupled system and to develop a synthesis technique for the realization of desired closed-loop pole configurations. Transfer matrix consequences of decoupling are examined and practical implications discussed through numerical examples.
\end{abstract}

\section{INTRODUCTION}

$\mathrm{T}$ YHE DEVELOPMENT of techniques for the design of multivariable control systems is of considerable practical importance. A particular design approach involves the use of feedback to achieve closed-loop control system stability. In conjunction with this approach, it is often of interest to know whether or not it is possible to have inputs control outputs independently, i.e., a single input influences a single output. This is, in heuristic terms, the problem of decoupling.

The problem of decoupling a time-invariant linear system using state variable feedback and the relation of this problem to control system design have been discussed by several authors. ${ }^{[1]-[3]}$ Morgan ${ }^{[1]}$ considered the question of decoupling for systems whose state equations had a somewhat special form. His main result, which is a special case of the main theorem of this paper, was the following: the time-invariant linear system

$$
\begin{aligned}
& \dot{x}=A x+B u \\
& y=C x
\end{aligned}
$$

can be decoupled if the matrix $C B$ is nonsingular. Rekasius ${ }^{[2]}$ extended Morgan's result and outlined an essentially trial-and-error procedure for specifying a certain number of the system's poles while decoupling the system. Neither Morgan nor Rekasius gave a clear

First part of manuscript received November 28, 1966; second part of manuscript received March 29, 1967. First part revised April 19, 1967; first and second parts combined and revised July 14, 1967. This research was supported in part by the Air Force Office of Scientific Research under AFOSR Grant 693-67 and in part by NASA.

P. L. Falb is with the Div. of Applied Mathematics, Brown University, Providence, R. I., and NASA Electronics Research Center, Cambridge, Mass.

IV. A. Wolovich is with NASA Electronics Research Center, Cambridge, Mass. proof of sufficiency, and they did not consider the question of necessity.

In this paper, a necessary and sufficient condition for decoupling will be given; a characterization of the class of feedback matrices which decouple a system will be determined; the number of closed-loop poles which can be specified while decoupling will also be determined; and a synthesis procedure for obtaining desired closedloop pole configurations will be developed. In line with these objectives, the remainder of this paper is divided in to the following sections:

II. Definitions

III. Main Theorem

IV. Class of Decoupling Matrices

V. A Synthesis Procedure

VI. Decoupling by Output Feedback

VII. A Practical Example.

In Section II precise definitions of state variable feedback and decoupling are given. Then the basic necessary and sufficient condition for decoupling is proved in Section III. Using the main theorem, a description of all the decoupling matrices is presented in Section IV. Next, in Section $V$ the questions of synthesis and closedloop pole placement are examined. In Section VI state variable feedback is replaced by output feedback and the relevant theory developed. The practical potential of the methods is indicated in the discussion of a V/STOL stability augmentation system in Section VII.

\section{Definitions}

Consider the time-invariant linear system

$$
\begin{aligned}
& \dot{x}=A x+B u \\
& y=C x
\end{aligned}
$$

where $x$ is an $n$ vector called the state, $u$ an $m$ vector called the control (or input), $y$ an $m$ vector called the output, and $A, B$, and $C$ are $n \times n, n \times m$, and $m \times n$ matrices, respectively. It is assumed that $m \leq n$. If $F$ is an $m \times n$ matrix and $G$ a nonsingular $m \times m$ matrix, then the substitution of

$$
u=F x+G \omega
$$

where $\omega$ represents the new $m$ vector control (Fig. 1), in to (1) shall be called linear state variable feedback.

Let $d_{1}, d_{2}, \cdots, d_{m}$ be given by

$$
d_{i}=\min \left\{j: C_{i} \boldsymbol{A}^{j} B \neq 0, \quad j=0,1, \cdots, n-1\right\}
$$


$\underline{\omega}$

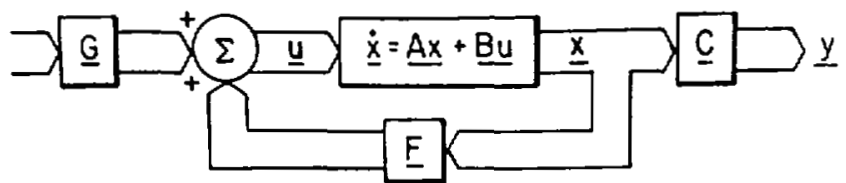

Fig. 1. Multivariable feedback system.

or

$$
d_{i}=n-1 \quad \text { if } C_{i} A^{j} B=0 \quad \text { for all } j
$$

where $C_{i}$ denotes the $i$ th row of $C$. Then, a simple calculation shows that

$$
\begin{array}{ll}
\mathrm{C}_{i}(A+B F)^{k}=C_{i} A^{k}, & k=0,1, \cdots, d_{i} \\
\mathrm{C}_{i}(A+B F)^{k}=C_{i} A^{d_{i}}(A+B F)^{k-d_{i}}, & k=d_{i}+1, \cdots, n
\end{array}
$$

for $i=1, \cdots, m$. Application of the state variable feedback (2) and repeated differentiation together with (4) yield the relations

$$
\begin{aligned}
& y_{i}=C_{i x}=C_{i}(A+B F)^{0} \mathrm{x} \\
& \dot{y}_{i} \quad=C_{i} A x=C_{i}(A+B F)_{x} \\
& \text { - } \quad \text {. } \\
& \dot{y}_{i}^{\left(d_{i}\right)}=C_{i} \dot{A}^{d_{i} \mathrm{X}}=C_{i}(\dot{A}+B F)^{d_{i X}} \\
& y_{i}^{\left(d_{i}+1\right)}=C_{i}(A+B F)^{d_{i}+1} \bar{x}+C_{i}(A+B F)^{d_{i}} B G \omega \\
& \text { - } \\
& \dot{y}_{i}^{(n)}=C_{i}(A+B F)^{n} \mathrm{x}+C_{i}(A+B F)^{n-1} B G \omega \\
& +\cdots+C_{i}(A+B F)^{d_{i}} B G \omega^{\left(n-d_{i}-1\right)}
\end{aligned}
$$

where $y_{i}, i=1, \cdots, m$, is the $i$ th component of $y$. In view of the Cayley-Hamilton theorem,

$$
(A+B F)^{n}=\sum_{k=0}^{n-1} p_{k}(F)(A+B F)^{k}
$$

where the $p_{k}(F)$ are scalars depending on $F$. Thus, $x$ can be eliminated from the final relation of (5) to give

$$
y_{i}{ }^{(n)}-\sum_{k=0}^{n-1} p_{k}(F) y_{i}{ }^{(k)}=\operatorname{tr}\left(L^{i}\{F, G\} \Omega\right)
$$

where $\operatorname{tr}(\cdot)$ denotes the trace of a matrix, $\Omega$ is the $m \times n$ matrix given by

$$
\boldsymbol{\Omega}=\left[\begin{array}{l|l|l|l}
\boldsymbol{\omega} & \boldsymbol{\omega}^{(1)} & \cdots & \boldsymbol{\omega}^{(n-1)}
\end{array}\right]
$$

and $L^{i}\{F, G\}$ is the $n \times m$ matrix given by where 0 is a zero matrix consistent with the order of $L^{i}\{F, G\}$. If $E_{i j}$ denotes the $m \times m$ matrix with 1 as $i j$ th entry and zeros elsewhere, then $E_{i 2} \mathbf{\Omega}$ is an $m \times n$ matrix with the $i$ th row identical to the $i$ th row of $\Omega$ and all other rows zero. The matrix $E_{i 2} \Omega$ will be denoted by $\mathbf{\Omega}^{i}$. The following definition can now be made.

The matrices $F$ and $G$, with $G$ nonsingular, decouple the system (1) if

$$
\begin{array}{r}
y_{i}^{(n)}-\sum_{k=0}^{n-1} p_{k}(F) y_{i}{ }^{(k)} \equiv \operatorname{tr}\left(L^{i}\{F, G\} \Omega\right) \\
\equiv \operatorname{tr}\left(L^{i}\{F, G\} \mathbf{\Omega}^{i}\right), \\
i=1, \cdots, m
\end{array}
$$

and if

$$
\operatorname{tr}\left(L^{i}\{F, G\} \mathbf{\Omega}\right) \neq 0, \quad i=1, \cdots, m .
$$

Note that this is a precise definition that does not involve vague statements about inputs controlling outputs independently.

\section{MAIN THEOREM}

With the definitions of Section II, it is now possible to state and prove a theorem which gives a necessary and sufficient condition for decoupling.

\section{Theorem 1}

Let $B^{*}$ be the $m \times m$ matrix given by

$$
B^{*}=\left[\begin{array}{c}
\mathrm{C}_{1} A^{d_{1}} B \\
\mathrm{C}_{2} A^{d_{2}} B \\
\vdots \\
C_{m} A^{d_{m}} B
\end{array}\right] .
$$

Then there is a pair of matrices $F$ and $G$ which decouple the system (1) if and only if

$$
\operatorname{det} B^{*} \neq 0
$$

i.e., if and only if $B^{*}$ is nonsingular.

Proof: Suppose first that $B^{*}$ is nonsingular. Then it is claimed that the pair

$$
\begin{aligned}
& F^{*}=-B^{*-1} A^{*} \\
& G^{*}=B^{*-1}
\end{aligned}
$$

where

$$
A^{*}=\left[\begin{array}{c}
C_{1} A^{d_{1}+1} \\
\vdots \\
C_{m} A^{d_{m}+1}
\end{array}\right]
$$

$$
L^{i}\{F, G\}=\left[\begin{array}{c}
C_{i}\left[(A+B F)^{n-1}-p_{n-1}(F)(A+B F)^{n-2}-\cdots-p_{d_{i}+1}(F)(A+B F)^{d_{i}}\right] B G \\
C_{i}\left[(A+B F)^{n-2}-p_{n-1}(F)(A+B F)^{n-3}-\cdots-p_{d_{i}+2}(F)(A+B F)^{d_{i}}\right] B G \\
\cdot \\
C_{i}\left[(A+B F)^{d_{i}}\right] B G \\
0
\end{array}\right]
$$


decouples (1). In view of (4),

$$
C_{i}\left(A+B F^{*}\right)^{d_{i}+1}=C_{i} A^{d_{i} \div 1}+C_{i} A^{d_{i}} B F^{*} .
$$

But $C_{i} A^{d_{i} B}$ is simply the $i$ th row of $B^{*}$ and so it follows that

$$
C_{i} A^{d_{i}} B F^{*}=-B_{i}{ }^{*} B^{*-1} A^{*}=-A_{i}{ }^{*}=-C_{i} A^{d_{i}+1}
$$

where $B_{i}{ }^{*}$ and $A_{i}{ }^{*}$ are the $i$ th rows of $B^{*}$ and $A^{*}$, respectively. Thus

$$
C_{i}\left(A+B F^{*}\right)^{d_{i}+k}=0
$$

for any positive integer $k$. In a similar way, it follows that

$$
C_{i}\left(A+B F^{*}\right)^{d_{i}} B B^{*-1}=B_{i}^{*} B^{*-1}
$$

and hence that

$$
L^{i}\left\{F^{*}, G^{*}\right\}=\left[\begin{array}{c}
-p_{d_{i}+1}\left(F^{*}\right) B_{i}{ }^{*} B^{*-1} \\
-p_{d_{i+2}+2}\left(F^{*}\right) B_{i}{ }^{*} B^{*-1} \\
\vdots \\
B_{i}^{*} B^{*-1} \\
0
\end{array}\right] .
$$

However, $B_{i}{ }^{*} B^{*-1}=\epsilon_{i}$, a row vector with 1 in the $i$ th place and zeros elsewhere and so

$$
\begin{aligned}
\operatorname{tr}\left(L^{i}\left\{F^{*}, G^{*}\right\} \boldsymbol{\Omega}\right)= & -p_{d_{i}+1}(F) \omega_{i}-p_{d_{i}+2}(F) \omega_{i}^{(1)}-\cdots \\
& +\omega_{i}^{\left(n-d_{i}-1\right)} \\
= & \operatorname{tr}\left(L^{i}\left\{F^{*}, G^{*}\right\} \boldsymbol{\Omega}^{i}\right) \neq 0 .
\end{aligned}
$$

In other words, $F^{*}$ and $G^{*}$ decouple (1).

Now suppose that there is a pair of matrices $F, G$ which decouple (1). Then it follows from (4) that

$$
C_{i}(A+B F)^{d_{i} B G}=B_{i}^{*} G, \quad i=1, \cdots, m .
$$

Since $C_{i} A^{i} B=0$ for all $j$ would imply that $\operatorname{tr}\left(L^{i}\{F, G\} \Omega\right)=0$, which would contradict the fact that $F$ and $G$ decouple (1), it is clear that $B_{i}{ }^{*} \neq 0$ for $i=1, \cdots, m$. As $G$ is nonsingular, $B_{i}{ }^{*} G \neq 0$ for all $i$. Since (10) is satisfied, it follows that $B_{i}{ }^{*} G$ is an $m$ row vector of the form $\alpha_{i} \varepsilon_{i}$ with $\alpha_{i} \neq 0$ [otherwise there would be $\omega_{j}{ }^{(k)}, j \neq i$, terms in $\left.\operatorname{tr}\left(L^{i}\{F, G\} \Omega\right)\right]$. Thus,

$$
B^{*} G=\left[\begin{array}{cccccc}
\alpha_{1} & & & & & 0 \\
& \alpha_{2} & & & & 0 \\
& & & \cdot & & \\
& & & & \cdot & \alpha_{m}
\end{array}\right]
$$

where

$$
\prod_{i=1}^{m} \alpha_{i} \neq 0 .
$$

Hence, $B^{*}$ is nonsingular since $G$ is.

The theorem just proved shows that $B^{*}$ is of paramount importance in the decoupling of (1) by state variable feedback. The basis for the choice of $F^{*}$ and $G^{*}$ in the proof of the theorem is the following observation.
Since (5) implies that

$$
y_{i}{ }^{\left(d_{i}+1\right)}=C_{i}(A+B F)^{d_{i}+1} x+C_{i} A^{d_{i}} B G \omega
$$

which may also be written in the form

$$
y^{*}=\left(A^{*}+B^{*} F\right) x+B^{*} G_{\omega}
$$

where $y^{*}$ is the $m$ vector with components $y_{i}^{\left(d_{i}+1\right)}$, it is clear that the choice $F=F^{*}$ and $G=G^{*}$ leads to

$$
y^{*}=\omega
$$

or, equivalently, to

$$
y_{i}^{\left(d_{i}+1\right)}=\omega_{i} .
$$

Caution: Equation (28) does not represent the decoupled system since, in general, it involves the cancellation of zeros. The equations of the decoupled system are given by $(10)$ or in state form as

$$
\begin{aligned}
& \dot{x}=(A+B F) x+B G_{\omega} \\
& y=C x
\end{aligned}
$$

where $F, G$ are a decoupling pair.

It has now been established that the nonsingularity of $B^{*}$ is a necessary and sufficient condition for the existence of a pair of matrices $F, G$ which decouple (1). In the next section, the set of all pairs $F, G$ which decouple (1) will be characterized under the assumption that $B^{*}$ is nonsingular. This characterization leads to "answers" to the following two questions:

1) the synthesis question, namely: how many closedloop poles can be specified for the decoupled system, how arbitrarily can they be specified, and how easily can an algorithm for specifying these poles be developed?

2) the output feedback question, namely: when can feedback of the form $\boldsymbol{u}=H y+G \omega$ decouple (1)?

\section{Class of Decolpling Matrices}

Let $F$ be an $m \times n$ matrix and let $G$ be a nonsingular $m \times m$ matrix. Under the assumption that (1) can be decoupled, necessary and sufficient conditions for $F, G$ to be a decoupling pair are determined in this section. These conditions turn out to be independent of $G$ so that it will make sense to speak of the class $\Phi$ of matrices $F$ which "decouple" (1).

\section{Definition}

Let $Q^{i}(F)$ be the $n \times m$ matrix given by

$$
Q^{i}(F)=\left[\begin{array}{c}
C_{i}(A+B F)^{n-1} B \\
C_{i}(A+B F)^{n-2} B \\
\vdots \\
C_{i}(\dot{A}+B F)^{d_{i} B} \\
0
\end{array}\right], \quad i=1, \cdots, m
$$

where 0 is a zero matrix consistent with the order of $Q^{i}(F)$. Let $P^{i}(F)$ be the $n \times n$ matrix given by 


$$
P^{i}(F)=\left[\begin{array}{cccc:c}
1 & -p_{n-1}(F) & \cdots & -p_{d_{i}+1}(F) & \\
0 & 1 & \cdots & -p_{d_{i}+2}(F) & 0 \\
\vdots & \vdots & \vdots & \vdots & \\
0 & 0 & \cdots & 1 & \\
\hdashline & \multicolumn{2}{c}{0} & & I
\end{array}\right] \text {, }
$$

where the $p_{k}(F)$ are the coefficients of the characteristic polynomial of $A+B F$, i.e.,

$$
(A+B F)^{n}=\sum_{0}^{n-1} p_{k}(F)(A+B F)^{k}
$$

and $I$ is an identity matrix consistent with the order of $P^{i}(F)$.

Since $P^{i}(F)$ is nonsingular, it follows that the rank of $P^{i}(F) Q^{i}(F)$ is the same as the rank of $Q^{i}(F)$. Note also that

$$
L^{i}\{F, G\}=P^{i}(F) Q^{i}(F) G
$$

where $L^{i}\{F, G\}$ is defined by (9). Thus

$$
\operatorname{rank}\left[L^{i}\{F, G\}\right]=\operatorname{rank}\left[Q^{i}(F)\right], \quad i=1, \cdots, m
$$

since $G$ is nonsingular. In view of the definition of decoupling, the following theorem can be established.

\section{Theorem 2}

If the pair $F, G$ decouples (1), then the rank of $Q^{i}(F)$ is one for all $i$; conversely, if the rank of $Q^{i}(F)$ is one for all $i$ and if $B^{*}$ is nonsingular, then the pair $F, B^{*-1}$ decouples the system (1).

Proof: Suppose first that $F, G$ decouple (1). Then

$$
\operatorname{tr}\left(L^{i}\{F, G\} \mathbf{\Omega}\right) \equiv \operatorname{tr}\left(L^{i}\{F, G\} \boldsymbol{\Omega}^{i}\right) \neq 0
$$

for all $i$ where $\Omega$ is the $m \times n$ matrix given by

$$
\boldsymbol{\Omega}=\left[\begin{array}{l|l|l|l|l}
\boldsymbol{\omega} & \boldsymbol{\omega} & \cdots & \boldsymbol{\omega}^{(n-1)}
\end{array}\right] .
$$

Since $\Omega$ is arbitrary, the $i$ th column of $L^{i}\{F, G\}$ is a nonzero vector, while every other column of $L^{i}\{F, G\}$ is the zero vector. It follows that $L^{i}\{F, G\}$ has rank one and hence, by (33), that $\operatorname{rank}\left[Q^{i}(F)\right]=1$.

Now suppose that rank $\left[Q^{i}(F)\right]=1$ for all $i$ and that $B^{*}$ is nonsingular. Since

$$
C_{i}(A+B F)^{d_{i} B}=C_{i} A^{d_{i} B}=B_{i}{ }^{*} \not \neq 0
$$

by the definition of $d_{i}$, where $B_{i}{ }^{*}$ is the $i$ th row of $B^{*}$, it follows that

$$
Q^{i}(F)=\left[\begin{array}{c}
\alpha_{1}{ }^{i} B_{i}{ }^{*} \\
\vdots \\
B_{i}{ }^{*} \\
0
\end{array}\right]
$$

and hence that

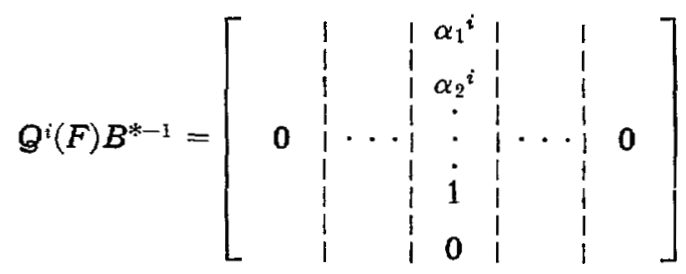

has only a nonzero $i$ th column. Thus,

$$
\begin{aligned}
\operatorname{tr}\left(L^{i}\left\{F, B^{*-1}\right\} \Omega\right) & =\operatorname{tr}\left(P^{i}(F) Q^{i}(F) B^{*-1} \Omega\right) \\
& =\operatorname{tr}\left(P^{i}(F) Q^{i}(F) B^{*-1} \Omega^{i}\right) \neq 0
\end{aligned}
$$

and so the pair $F, B^{*-1}$ decouples (1).

\section{Corollary 1}

If the pair $F$, $G$ decouples (1), then there is a diagonal matrix $\mathbf{\Lambda}$ such that $G=\mathbf{\Lambda} B^{*-1}$.

Proof: If $F, G$ decoupless (1), then $Q^{i}(F)$ is given by (37) and

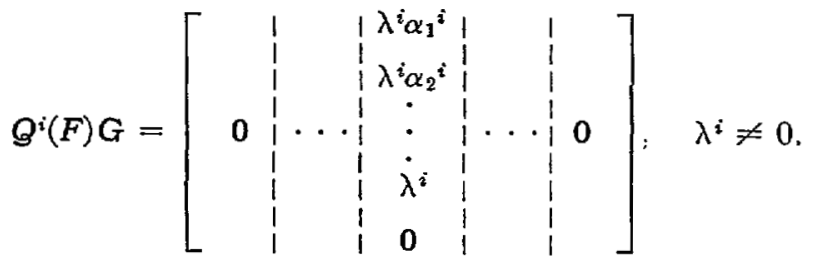

It follows that $B^{*} G=\operatorname{diag}\left[\lambda^{1}, \cdots, \lambda^{n}\right]$, and the corollary is established.

\section{Corollary 2}

If the pair $F, G$ decouples (1), then there is a diagonal matrix $\mathfrak{n}$ such that

$$
F B=B^{*-1}\left\{\mathrm{n} A^{* *}-A^{*}\right\} B
$$

where $A^{* *}$ and $A^{*}$ are given by

$$
A^{* *}=\left[\begin{array}{c}
C_{1} A^{d_{1}} \\
\vdots \\
C_{m} A^{d_{m}}
\end{array}\right], \quad A^{*}=A^{* *} A .
$$

Proof: The corollary is an immediate consequence of the relations

$$
\begin{aligned}
& C_{i}(A+B F)^{d_{i}+1}=C_{i} A^{d_{i}} B+C_{i} A^{d_{i}+1} B F B \\
& C_{i}(A+B F)^{d_{i}+1}=\gamma_{i} C_{i}(A+B F)^{d_{i}} B .
\end{aligned}
$$

In summary, thus far it has been shown that the nonsingularity of $B^{*}$ is a necessary and sufficient condition for the existence of a decoupling pair $F, G$. Furthermore, the set of all pairs $F, G$ which decouple (1) consists of matrices $F$ such that rank $\left[Q^{i}(F)\right]=1$ for all $i$, and $G$ such that $G=\mathbf{\Lambda} B^{*-1}$, where $\boldsymbol{\Lambda}$ is diagonal and nonsingular. In order to clarify these points, an example will now be presented. 


\section{Example 1}

Let

$$
\begin{gathered}
A=\left[\begin{array}{lll}
1 & 1 & 0 \\
0 & 2 & 0 \\
0 & 1 & 3
\end{array}\right], \quad B=\left[\begin{array}{rr}
1 & 1 \\
-1 & 1 \\
0 & 0
\end{array}\right], \\
C=\left[\begin{array}{lll}
1 & 0 & 0 \\
0 & 0 & 1
\end{array}\right] .
\end{gathered}
$$

Then

$$
B^{*}=\left[\begin{array}{l}
C_{1} B \\
C_{2} A B
\end{array}\right]=\left[\begin{array}{rr}
1 & 1 \\
-1 & 1
\end{array}\right]
$$

Thus, $B^{*}$ is nonsingular and the system can be decoupled. The set $\Phi$ of all $F$ which decouple the system (45) can now be obtained by determining all $2 \times 3$ matrices $F$ such that rank $\left[Q^{i}(F)\right]=1$. In this example, this implies that the elements of $\Phi$ must be of the form

$$
F=\left[\begin{array}{ccc}
f_{11} & f_{12} & f_{13} \\
f_{11} & -f_{12}-1 & -f_{13}^{\prime}
\end{array}\right] .
$$

\section{A Synthesis Procedure}

Theorem 2 does provide a procedure for determining $\Phi$, the class of all feedback matrices $F$ which decouple (1). However, the direct application of the condition, rank $\left[Q^{i}(F)\right]=1$ for all $i$, results only in constraints being placed on certain of the $m n$ parameters of $F$. What is still required is a procedure for specifying closed-loop system poles while simultaneously decoupling (1) using an appropriate $F \in \Phi$. In this light, a synthesis procedure will now be presented for directly obtaining a feedback matrix $F \in \Phi$ whose parameters are so determined as to yield a desired closed-loop pole structure.

In particular, suppose that $M_{k}, k=0,1, \cdots, \delta$ are given $m \times m$ matrices. Then the choice

$$
\begin{aligned}
& F=B^{*-1}\left[\sum_{k=0}^{\delta} M_{k} C A^{k}-A^{*}\right] \\
& G=B^{*-1}
\end{aligned}
$$

will, by (26), lead to

$$
y^{*}=\sum_{k=0}^{\delta} M_{k} C A^{k} \text { X }+\omega .
$$

If $\delta=\max d_{i}$ and the $M_{k}$ are suitably chosen, i.e. $\boldsymbol{M}_{k}=\operatorname{diag}\left[m_{k}{ }^{1}, m_{k}{ }^{2}, \cdots, m_{k}{ }^{m}\right], i=1,2, \cdots, m$, then (30) may be written in the form

$$
y^{*}=\sum_{k=0}^{\delta} M_{k} y^{(k)}+\omega
$$

or

$$
\begin{aligned}
y_{i}{ }^{\left(d_{i}+1\right)}=m_{0}{ }^{i} y_{i}+m_{1}{ }^{i} y_{i}{ }^{(1)}+\cdots+m_{d_{i}}{ }^{i} y_{i}{ }^{\left(d_{i}\right)}+\omega_{i}, \\
i=1,2, \cdots, m
\end{aligned}
$$

which indicates that $F$ and $G$ decouple (1) and that $m+\sum_{i=1}^{m} d_{i}$ of the closed-loop poles can be varied by varying the $\boldsymbol{M}_{k}$. In this light, consider the following example.

\section{Example 2}

Let

$$
\begin{gathered}
A=\left[\begin{array}{rrrrrr}
0 & 1 & 0 & 0 & 0 & 0 \\
0 & 0 & 1 & 0 & 0 & 0 \\
0 & 0 & -1 & 0 & 0 & 0 \\
0 & 0 & 0 & 0 & 1 & 0 \\
0 & 0 & 0 & 0 & 0 & 1 \\
0 & 0 & 0 & 0 & 0 & 0
\end{array}\right], \quad B=\left[\begin{array}{ll}
1 & 0 \\
0 & 0 \\
1 & 0 \\
0 & 0 \\
0 & 0 \\
0 & 0 \\
1 & 0
\end{array}\right], \\
C^{\prime}=\left[\begin{array}{ll}
0 \\
0 & 1 \\
0 & 0 \\
0 & 0
\end{array}\right] .
\end{gathered}
$$

Now

$$
B^{*}=\left[\begin{array}{ll}
0 & 1 \\
1 & 0
\end{array}\right], \quad A^{*}=\left[\begin{array}{llllll}
0 & 1 & 0 & 0 & 0 & 0 \\
0 & 0 & 0 & 0 & 0 & 0
\end{array}\right] .
$$

Since $B^{*}$ is nonsingular, the system can be decoupled. Setting, for example,

$$
M_{0}=M_{1}=M_{2}=\left[\begin{array}{ll}
0 & 0 \\
0 & 1
\end{array}\right]
$$

one obtains, using (29), the decoupled system

$$
\begin{aligned}
y_{1}{ }^{(6)}= & y_{1}{ }^{(2)}+2 y_{1}{ }^{(3)}+2 y_{1}{ }^{(4)}-\omega_{1}{ }^{(1)}-2 \omega_{1}{ }^{(2)} \\
& -2 \omega_{1}{ }^{(3)}+\omega_{1}{ }^{(5)} \\
y_{2}{ }^{(6)}= & y_{2}{ }^{(2)}+2 y_{2}{ }^{(3)}+2 y_{2}{ }^{(4)}+\omega_{2}{ }^{(2)}+\omega_{2}{ }^{(3)} .
\end{aligned}
$$

Note that in this case

$$
\operatorname{det}(s I-A-B F)=s^{2}(s+1)\left(s^{3}-s^{2}-s-1\right)
$$

where the poles representing $s\left(s^{3}-s^{2}-s-1\right)$ have been specified by the choice of the $M_{k}$. Other choices of the $M_{k}$ would lead to other closed-loop pole configurations. Therefore, if $B^{*}$ is nonsingular, $m+\sum_{i=1}^{m} d_{i}$ of the system's closed-loop poles can be arbitrarily specified $\left(d_{i}+1\right.$ at a time) while simultaneously decoupling the system using the synthesis procedure. The synthesis question is, therefore, partially resolved, although some points still require clarification. In particular, it will be shown that $m+\sum_{i=1}^{m} d_{i}$ can never exceed $n$, the number of system poles, and that it is sometimes possible to specify more than $m+\sum_{i=1}^{m} d_{i}$ poles while simul taneously decoupling the system. 


\section{Lemma}

Let $K$ be the $\left(m+\sum_{i=1}^{m} d_{i}\right) \times n$ matrix given by

$$
K=\left[\begin{array}{l}
C_{1} \\
\vdots \\
\dot{C}_{1} A^{d_{1}} \\
C_{2} \\
\vdots \\
\dot{C}_{2} A^{d_{2}} \\
\vdots \\
\dot{C}_{m} A^{d_{m}}
\end{array}\right] .
$$

Then rank $[K]=m+\sum_{i=1}^{n} d_{i}$ and hence $m+\sum_{i=1}^{m} d_{i} \leq n$.

Proof: Let $k_{i}$ denote the $i$ th row of $K$, and let $r_{i}$ be arbitrary scalars such that

$$
\sum_{1}^{v} r_{i} k_{i}=0
$$

where

$$
\nu=m+\sum_{1}^{\mathfrak{m}} d_{i}
$$

In order to establish the lemma, one need only show that (57) implies that each $r_{i}=0$. However, this follows directly from (57) by successive postmultiplication by $B, A B, \cdots, A^{\delta} B$, and the fact that $B^{*}$ is nonsingular.

$$
\left[\frac{\left(s-f_{24}\right) \cdot}{\left(s-f_{24}\right)\left(s^{3}-f_{13} s^{2}-f_{12} s-f_{11}\right)}\right.
$$

Now let $p$ denote the number of closed-loop poles which can be specified while decoupling, and let $f$ denote the number of free parameters (entries) in a decoupling matrix $F$ [for example, $f=3$ in (47)]. Then the lemma and (51) combine to give

$$
\begin{aligned}
& m+\sum_{1}^{m} d_{i} \leq p \leq n \\
& m+\sum_{1}^{m} d_{i} \leq f .
\end{aligned}
$$

Moreover, if $m+\sum_{1}^{m} d_{i}=n$, then all $n$ of the closedloop poles can be arbitrarily positioned while simultaneously decoupling the system. Also, if $f=m+\sum_{1}^{m} d_{i}$, then (51) gives direct physical significance to the free parameters in $F$. If $f>m+\sum_{1}^{m} d_{i}$ (or $n$ ), then it may be possible to specify more than $m+\sum_{1}^{m} d_{i}$ of the closed-loop poles. In this situation, it is often advantageous to calculate $C(s I-A-B F)^{-1} B B^{*-1}$ with $f$ entries in $F$ remaining arbitrary. The following examples illustrate these ideas and some of the difficulties involved in their application.

\section{Example 3}

Let

$$
\begin{gathered}
A=\left[\begin{array}{llll}
0 & 1 & 0 & 0 \\
0 & 0 & 1 & 0 \\
0 & 0 & 0 & 0 \\
0 & 0 & 0 & 1
\end{array}\right], \quad B=\left[\begin{array}{ll}
0 & 0 \\
0 & 0 \\
1 & 0 \\
0 & 1
\end{array}\right], \\
C^{\prime}=\left[\begin{array}{ll}
1 & 0 \\
0 & 0 \\
0 & 0 \\
0 & 1
\end{array}\right] .
\end{gathered}
$$

Then

$$
B^{*}=\left[\begin{array}{l}
C_{1} A^{2} B \\
C_{2} B
\end{array}\right]=I
$$

and $m+\sum_{1}^{m} d_{i}=4=n$. Thus, all the closed-loop poles can be specified by using the synthesis procedure.

$$
F=\left[\begin{array}{llll}
f_{11} & f_{12} & f_{13} & 0 \\
0 & 0 & 0 & f_{24}
\end{array}\right]
$$

Moreover, application of Theorem 2 shows that (62) represents the most general form for a decoupling $F$ so that $f=4=n$. The general form of the decoupled transfer matrix is

$$
\left.\begin{array}{c}
0 \\
\frac{\left(s^{3}-f_{13} s^{2}-f_{12} s-f_{11}\right)}{\left(s-f_{24}\right)\left(s^{3}-f_{13} s^{2}-f_{12} s-f_{11}\right)}
\end{array}\right] .
$$

\section{Example 4}

Let

$$
\begin{gathered}
A=\left[\begin{array}{lll}
0 & 1 & 0 \\
2 & 3 & 0 \\
1 & 1 & 1
\end{array}\right], \quad B=\left[\begin{array}{ll}
0 & 0 \\
1 & 0 \\
0 & 1
\end{array}\right], \\
C=\left[\begin{array}{lll}
1 & 1 & 0 \\
0 & 0 & 1
\end{array}\right] .
\end{gathered}
$$

Then

$$
B^{*}=\left[\begin{array}{l}
C_{1} B \\
C_{2} B
\end{array}\right]=I .
$$

Thus, $B^{*}$ is nonsingular and the system (64) can be decoupled. It can be shown that the elements of $\Phi$ must be such that

$$
F=\left[\begin{array}{ccc}
f_{11} & f_{12} & 0 \\
-1 & -1 & f_{23}
\end{array}\right]
$$

so that $f=n=3>2=m+\sum_{1}^{m} d_{i}$. Moreover, the closedloop transfer matrix is given by 


$$
\begin{aligned}
& C(s I-A-B F)^{-1} B B^{*-1} \\
&= \frac{\left[\begin{array}{cc}
(s+1)\left(s-f_{23}-1\right) & 0 \\
0 & s^{2}-\left(f_{12}+3\right) s-\left(f_{11}+2\right)
\end{array}\right]}{\left(s-f_{23}-1\right)\left[s^{2}-\left(f_{12}+3\right) s-\left(f_{11}+2\right)\right]}
\end{aligned}
$$

so that all of the closed-loop poles can be specified by application of Theorem 2. However, note that application of the synthesis procedure in this case would allow one to specify only two of the three closed-loop poles.

\section{Decotpling by Oltplt Feedback}

Since output feedback is only a special case of state variable feedback, i.e.,

$$
u=H y+G \omega \equiv H C x+G \omega
$$

with $H C$ replacing $F$, it follows immediately that (1) can be decoupled using output feedback if and only if 1) $B^{*}$ is nonsingular and 2) there is an $m \times m$ matrix $H$ such that rank $\left[Q^{i}(H C)\right]=1$ for $i=1, \cdots, m$. These conditions provide a suitable test for whether or not a system can be decoupled using output feedback.

\section{Example 5}

Let

$$
\begin{gathered}
A=\left[\begin{array}{lll}
1 & 1 & 0 \\
0 & 2 & 0 \\
0 & 1 & 3
\end{array}\right], \quad B=\left[\begin{array}{rr}
1 & 1 \\
-1 & 1 \\
0 & 0
\end{array}\right] \\
C=\left[\begin{array}{lll}
1 & 0 & 0 \\
0 & 0 & 1
\end{array}\right] .
\end{gathered}
$$

Then

$$
B^{*}=\left[\begin{array}{rr}
1 & 1 \\
-1 & 1
\end{array}\right]
$$

is nonsingular so that the system defined by (69) can be decoupled. However, it is not possible to decouple this system using output feedback. To see this, observe that Theorem 2 and (39) imply that an $F$ which decouples must be of the form

$$
F=\left[\begin{array}{ccc}
f_{11} & f_{12} & f_{13} \\
f_{11} & -f_{12}-1 & -f_{13}
\end{array}\right]
$$

and that $H C$ must be of the form

$$
H C=\left[\begin{array}{lll}
h_{11} & 0 & h_{12} \\
h_{21} & 0 & h_{22}
\end{array}\right] \text {. }
$$

Equations (71) and (72) lead to the contradictory requirement that $f_{12}=0$ and $f_{12}=-1$. This example illustrates the point that decoupling by state variable feedback need not imply decoupling by output feedback.

It should be noted that, although a system may be decoupled using output feedback, some of the flexibility of specifying closed-loop poles, as with state variable feedback, will in general be lost. For example, consider the system described by (60), with the most general $H$ given by

$$
H=\left[\begin{array}{ll}
h_{11} & 0 \\
0 & h_{22}
\end{array}\right] .
$$

Since $\operatorname{det}(s I-A-B H C)=\left(s-1-h_{22}\right)\left(s^{3}-h_{11}\right)$, output feedback will not be adequate to stabilize the system although state variable feedback provides a higher degree of flexibility (63).

\section{Example 6}

Consider the system described by (64). It has been shown in (67) that state variable feedback can be used to decouple the system while simultaneously specifying all three closed-loop poles. Application of Theorem 2 and (39) imply that any $2 \times 2$ matrix $H$ of the form

$$
H=\left[\begin{array}{cc}
h_{11} & 0 \\
-1 & h_{22}
\end{array}\right]
$$

will define an output feedback which decouples this system. From (74) it follows that

$\operatorname{det}(s I-A-B H C)=\left(s-1-h_{22}\right)\left(s^{2}-\left(h_{11}+3\right) s-\left(h_{11}+2\right)\right)$

and hence that the system can be stabilized using output feedback (e.g., $h_{22}=-1, h_{11}=-5$ ), although the poles are not completely arbitrary.

\section{Example 7}

Let

$$
\begin{gathered}
A=\left[\begin{array}{lll}
1 & 1 & 0 \\
0 & 1 & 0 \\
0 & 0 & 1
\end{array}\right], \quad B=\left[\begin{array}{ll}
0 & 1 \\
1 & 0 \\
1 & 0
\end{array}\right], \\
C^{\prime}=\left[\begin{array}{rr}
1 & 0 \\
1 & 1 \\
-1 & 0
\end{array}\right] .
\end{gathered}
$$

Then

$$
\begin{gathered}
B^{*}=C B=\left[\begin{array}{ll}
0 & 1 \\
1 & 0
\end{array}\right] \\
m+\sum_{1}^{m} d_{i}=2<3=n .
\end{gathered}
$$

It can be shown, using Theorem 2, that any decoupling $F$ is of the form

$$
F=\left[\begin{array}{lrr}
0 & f_{12} & f_{13} \\
f_{21} & f_{22} & -1-f_{22}
\end{array}\right]
$$

so that $f=4$. However, the closed-loop transfer matrix is given by

$$
\frac{\left[\begin{array}{cc}
(s-1)\left(s-f_{12}-f_{13}-1\right) & 0 \\
0 & (s-1)\left(s-f_{21}-1\right)
\end{array}\right]}{(s-1)\left(s-f_{21}-1\right)\left(s-f_{12}-f_{13}-1\right)}
$$


so that $p=2$, i.e., only two of the closed-loop poles can be specified. It can also be shown for this example that output feedback leads to the transfer matrix

$$
\frac{\left[\begin{array}{cc}
(s-1)\left(s-h_{12}-1\right) & 0 \\
0 & (s-1)\left(s-h_{21}-1\right)
\end{array}\right]}{(s-1)\left(s-h_{12}-1\right)\left(s-h_{21}-1\right)}
$$

so that output and state variable feedback are equivalent. This, as previous examples illustrate, is not true in general.

\section{A Practical Exayple}

An area in which decoupling techniques may be of interest is the design of flight control and stability augmentation systems. Consider, for example, the following linearized longitudinal equations of motion for a lift-fan $V / S T O L$ vehicle ${ }^{1}$ in a hovering condition.

$$
\begin{aligned}
{\left[\begin{array}{c}
\dot{u} \\
\dot{\theta} \\
\ddot{\theta} \\
\dot{w} \\
\Delta \dot{x} \\
\Delta \dot{z}
\end{array}\right]=} & {\left[\begin{array}{llllll}
X_{u} & X_{\theta} & 0 & 0 & 0 & 0 \\
0 & 0 & 1 & 0 & 0 & 0 \\
M_{u} & 0 & M_{\dot{\theta}} & M_{w} & 0 & 0 \\
0 & 0 & Z_{\theta} & Z_{w} & 0 & 0 \\
1 & 0 & 0 & 0 & 0 & 0 \\
0 & 0 & 0 & 1 & 0 & 0
\end{array}\right] } \\
& +\left[\begin{array}{lll}
X_{c v} & 0 & 0 \\
0 & 0 & 0 \\
M_{c v} & M_{\theta n f} & 0 \\
0 & Z_{\theta} & Z_{c s} \\
0 & 0 & 0 \\
0 & 0 & 0
\end{array}\right]\left[\begin{array}{c}
\delta_{v} \\
\delta_{n f} \\
\delta_{s}
\end{array}\right]
\end{aligned}
$$

where

$$
\begin{aligned}
u & =\text { incremental longitudinal }(x) \text { velocity change } \\
\theta & =\text { incremental pitch angle } \\
\dot{\theta} & =\text { pitch rate, } \\
w & =\text { incremental vertical }(z) \text { velocity change, } \\
\Delta x & =\text { incremental position error } \\
\Delta z & =\text { incremental altitude error } \\
\delta_{v} & =\text { incremental collective fan input } \\
\delta_{n f} & =\text { incremental nose fan input } \\
\delta_{s} & =\text { incremental fan stagger input. }
\end{aligned}
$$

The relevant outputs in this example are $\theta, \Delta x$, and $\Delta z$, and the subscripted capitals (e.g., $X_{u}$ ) are the relevant stability derivatives.

The output matrix $\mathbf{C}$ is thus defined as

$$
C=\left[\begin{array}{llllll}
0 & 1 & 0 & 0 & 0 & 0 \\
0 & 0 & 0 & 0 & 1 & 0 \\
0 & 0 & 0 & 0 & 0 & 1
\end{array}\right]
$$

\footnotetext{
1 Similar to the XV-5A.
}

In this example

$$
B^{*}=\left[\begin{array}{l}
C_{1} A B \\
C_{2} A B \\
C_{3} A B
\end{array}\right]=\left[\begin{array}{lll}
M_{c v} & M_{\theta n f} & 0 \\
X_{c v} & 0 & 0 \\
0 & Z_{\theta} & Z_{c s}
\end{array}\right]
$$

and is nonsingular since it is assumed that $Z_{c s} M_{\theta_{n j}} X_{c o} \neq 0$. Therefore

$$
m+\sum_{1}^{3} d_{i}=6
$$

and hence all six of the closed-loop poles can be arbitrarily specified while simultaneously decoupling this system. It can be shown, using Theorem 2, that a decoupling $F$ has 6 (i.e., $f=6$ ) free parameters. Thus, the synthesis procedure (Section $V$ ) can be directly applied to give physical significance to these free parameters. For example, suppose that independent pitch, translation, and altitude control is desired, i.e.,

$$
\begin{aligned}
\ddot{\theta} & =m_{1}{ }^{0} \theta+m_{1}{ }^{1} \dot{\theta}+\omega_{1} \\
\Delta \ddot{x} & =m_{2}{ }^{0} \Delta x+m_{2}{ }^{1} \Delta \dot{x}+\omega_{2} \\
\Delta \ddot{z} & =m_{3}{ }^{0} \Delta z+m_{3}{ }^{1} \Delta \dot{z}+\omega_{3} .
\end{aligned}
$$

According to the synthesis procedure, $F$ can be set equal to

$$
B^{*-1}\left[\sum_{0}^{1} M_{k} C A^{k}-A^{*}\right] .
$$

It can be shown that for this decoupling $F$,

$$
A+B F=\left[\begin{array}{llllll}
m_{2}{ }^{1} & 0 & 0 & 0 & m_{2}{ }^{0} & 0 \\
0 & 0 & 1 & 0 & 0 & 0 \\
0 & m_{1}{ }^{0} & m_{1}{ }^{1} & 0 & 0 & 0 \\
0 & 0 & 0 & m_{3}{ }^{1} & 0 & m_{3}{ }^{0} \\
1 & 0 & 0 & 0 & 0 & 0 \\
0 & 0 & 0 & 1 & 0 & 0
\end{array}\right] .
$$

If $G$ is now set equal to $B^{*-1}$, the closed-loop transfer matrix is

$$
\begin{aligned}
& C(s I-A-B F)^{-1} B B^{*-1} \\
& =\frac{\left[\begin{array}{ccc}
\left(s^{2}-m_{2}{ }^{1} s-m_{2}{ }^{0}\right)\left(s^{2}-m_{3}{ }^{1} s-m_{3}{ }^{0}\right), & 0, & 0 \\
0, & \left(s^{2}-m_{1}{ }^{1} s-m_{1}{ }^{0}\right)\left(s^{2}-m_{3}{ }^{1}-m_{3}{ }^{0}\right), & 0 \\
0, & 0, & \left(s^{2}-m_{1}{ }^{1} s-m_{1} 0\right)\left(s^{2}-m_{2}{ }^{1}-m_{2}{ }^{0}\right)
\end{array}\right]}{\left(s^{2}-m_{1}{ }^{1} s-m_{1}\right)\left(s^{2}-m_{2}{ }^{1}-m_{2}{ }^{0}\right)\left(s^{2}-m_{3}{ }^{1} s-m_{3}\right)} .
\end{aligned}
$$

If the $m_{j}{ }^{i}$ are suitably chosen, then, in effect, the pilot will be faced with the task of controlling three highly stable second-order systems. This example serves only to indicate a potential practical area of application for the ideas presented in this paper.

The above examples illustrate the techniques developed for synthesizing decoupling controllers for multivariable systems. 


\section{Conclusions}

The problem of decoupling a time-invariant linear system using state variable feedback has been considered. Necessary and sufficient conditions for decoupling have been determined in terms of the nonsingularity of a matrix $B^{*}$. The class $\Phi$ of all feedback matrices which decouple a system has been characterized, and a synthesis technique for the realization of desired closed-loop pole configurations has been developed. In essence, the major theoretical questions relating to decoupling via state variable feedback have been resolved for time-invariant linear systems.

A number of interesting potential areas of future research arise from the results obtained here. In particular, the question of extending the theory to the timevarying situation is of considerable interest. Some preliminary results relating to stabilization have already been obtained. ${ }^{[6]}$ The design of aircraft and V/STOL stability augmentation systems via decoupling techniques is a potential practical area of application as was mentioned in Section VII. Practical implementation of the techniques presented in this paper has begun but much remains to be done before the theory is transformed into a practical design technique.

\section{REFERENCES}

${ }^{[1]}$ B. S. Morgan, "The synthesis of linear multivariable systems by state variable feedback," Preprints, Joint Automatic Control Conf. (Stanford, Calif., June 1964), pp. 468-472. Also IEEE Trans. A utomatic Control, yol. AC-9, pp. 405-411, October 1964.

[2] Z. V. Rekasius, "Decoupling of multivariable systems by means of state variable feedback," Proc. 3rd Ann. Allerton Conf. on Circuit and System Theory (Urbana, Ill., 1965), pp. 439-447.

[3] P. L. Falb and W. A. Wolovich, "On the decoupling of multivariable systems," Preprints, Joint Automatic Control Conf. (Philadelphia, Pa., June 1967), pp. 791-796.

[4] L. A. Zadeh and C. A. Desoer, Linear Syslem. Theory. New York: McGraw-Hill, 1963.

${ }^{[5]}$ E. Seckel, Stability and Control of Airplanes and Helicopters. New York: Academic Press, 1964.

[6] W. A. Wolovich, "On the stabilization of controllable systems," IEEE Trans. Automatic Control (to be published).

Peter L. Falb (M'64) was born in New York, N. Y., on July 26, 1936. He received the A.B., M.A., and Ph.D. degrees, all in mathematics, from Harvard University, Cambridge, Mass., in 1956, 1957, and 1961, respectively.

From 1960 to 1965 he was at the M.I.T. Lincoln Laboratory, Lexington, Mass. In 1965 he was a Visiting

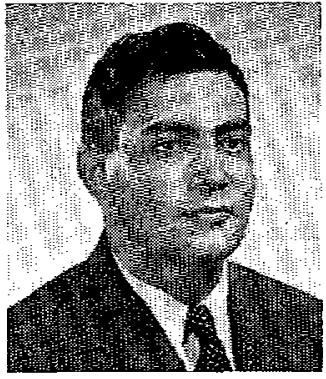

Associate Professor in the Department of Aeronautics and Astronautics at Stanford University, Stanford, Calif. In 1966 he was Associate Professor of Information and Control at the University of Michigan, Ann Arbor. Currently he is Associate Professor of Applied Mathematics at Brown University, Providence, R. I., and a Consultant for NASA and Bolt Beranek and Newman, Inc. His research interests are in control and applied mathematics, and he is coauthor of the book Optimal Control (McGraw-Hill, 1966). He is a Member of the Editorial Board of the SIAM Journal on Control.

Dr. Falb is a member of Phi Beta Kappa, Sigma Xi, the American Mathematical Society, and the Society for Industrial and Applied Mathematics.

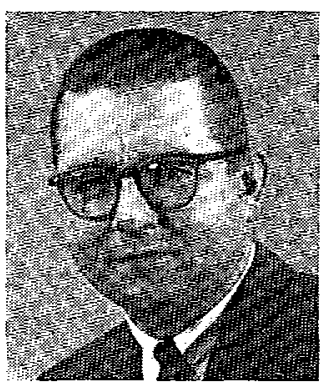

William A. Wolovich (.',64) was born in Hartford, Conn., on October 15, 1937. He received the B.S.E.E. degree from the University of Connecticut, Storrs, in 1959, and the M.S.E.E. degree from Worcester Polytechnic Institute, Worcester. Mass., in 1961.

From 1959 to 1961 he was with the Research Laboratories of the United Aircraft Corp. He served as Ground Electronics Officer in the U. S. Air Force from 1961 to 1964. In 1964 he joined the staff of the Electronics Research Center in the Control and Information Systems Laboratory. He has taken graduate courses at the Massachusetts Institute of Technology, Cambridge, and Northeastern University, Boston, Mass., and he is currently a graduate student in the Department of Engineering, Brown University, Providence, R. I., working in the area of dynamic systems theory.

Mr. Wolovich is a member of Tau Beta Pi and Eta Kappa Nu. 